\title{
Safety and Feasibility of Autologous Mesenchymal Stem Cell Transplantation in Chronic Stroke in Indian patients. A four-year follow up
}

\author{
Bhasin $\mathrm{A}^{1}$, Kumaran $\mathrm{SS}^{2}$, Bhatia $\mathrm{R}^{1}$, Mohanty $\mathrm{S}^{3}$, Srivastava MVP
}

\begin{abstract}
Introduction: Stem cell (SC) therapy has been envisioned as a therapeutic vehicle to promote recovery in resistant neurological diseases. Knowing the logistics and paradigms in recovery processes after Stroke, clinicians have pioneered the transplantation therapy. This study presents four-year follow up of our previous trial transplanting bone-marrow-derived animal-free culture expanded intravenous mesenchymal stem cells (MSCs) in chronic stroke which was published in 2010.
\end{abstract}

Methods: We performed an open-label, pilot trial on 12 patients with chronic stroke. Patients were allocated to two groups, those who received intravenous autologous ex vivo cultured mesenchymal stem cells (MSC group) or those who did not (control group), all followed for four years from the day of cell transplantation.

Results: The reports have been optimistic regarding safety as we did not find any cell related side effects / mortality till 208th week. We observed that modified Barthel Index showed statistical significant improvement at 156 and 208 weeks of transplantation (95 \% CI : -10.27 to $0.07 ; \mathrm{p}=0.041$ ) follow up in the MSC group as compared to controls. The 2 nd and 3rd quartile for mBI in MSC group was $89 \& 90$ respectively suggesting good performance of patients in the stem cell group. The impairment scales i.e., Fugl Meyer, Ashworth tone scale, strength of hand muscles (MRC) did not show any significant improvement at 208th week which is similar to our previous published report.

Conclusion: This follow up study primarily indicates safety, tolerance and applicability of autologous mesenchymal stem cells in Stroke. MSCs may act as "chaperones" or work through paracrine mechanisms leading to functional recovery post stroke.

Key Words: Stem cell transplantation, Stroke, Recovery

\section{Introduction}

The knowledge and use of stem cells in regenerative medicine and drug development has been a soulful interest in biomedical field $^{[1]}$. Evidence of neurogenesis in the adult brain is well proven refuting the dilemma regarding CNS regeneration. With an enthralling research opportunities in medicine targeting treatment opportunities, cell transplantation and gene therapies act as panacea to improve the quality and expectancy of life in resistant neurological disorders. Stroke is associated with high mortality and severe morbidity and reports suggest that $50 \%$ stroke survivors suffer residual neurological deficits ${ }^{[2,3]}$. Because of the changed morbidity spectrum, the focus of interest is shifting towards behavioral recovery, although rehabilitation motor therapy is important for maximization of functional recovery after stroke $^{[4,5]}$. Stem cell therapy recently has been divided under two groups; "replacement therapy" and "neurotrophic therapy". Clinical trials of differentiated tumor cell lines, neural progenitor cells from primordial porcine striatum, and autologous bone marrow-derived mesenchymal stem cells (MSCs) have been conducted $^{[6]}$.
Neurorestorative processes contributing to functional improvement after chronic stroke include neurogenesis, angiogenesis, and synaptic plasticity. Cell transplantation is a cellular approach that has the potential to induce all of the neurorestorative processes essential for facilitating recovery of neurological function. Bone marrow-derived mesenchymal stem cells (MSCs) have great potential as therapeutic agents in stroke management, since they are easily accessible and can be rapidly expanded ex vivo for autologous transplantation. Increasing evidence suggests that bone marrow cells migrate throughout the brain and differentiate into neurons and glial cells ${ }^{[7-9]}$.

\section{MSC as neurorestorative therapy: Cognizance from our last trial}

The latest consensus reported improvement of stroke outcomes after systemic stem cell injection which relies on the paracrine / autocrine hypothesis of these cells i.e, non-cell-autonomous properties, release of relevant trophic factors, rather than on engraftment into the lesioned area. However, several variables related to the optimal patient, such as age, type of stroke, location size of the lesion, and timing of treatment, remain to be 
addressed $^{[10,11]}$. MSCs are excellent candidates for cell transplantation as they are easily accessible, and can be preserved with minimal loss of potency. Transplantation by these cells have been safe and has been widely used in clinical trials (NCT 01714167) of neurological, cardiovascular and immunological diseases with encouraging results $^{[12]}$. Our last report on MSC infusion in six chronic stroke patients established the safety and tolerance of MSC derived using serum-free media for expansion unlike bovine serum ${ }^{[13]}$. There was no significant difference in baseline clinical and radiological scores between the MSC and control groups, suggesting that the two groups were comparable to study the effectiveness of therapy after 8 and 24 weeks. There was no significant difference in FM and $\mathrm{mBI}$ scores after therapy ( 8 weeks: $\mathrm{p}=0.87, \mathrm{t}=0.161$ and $\mathrm{p}=0.95, \mathrm{t}=0.065$, respectively; and at follow-up ( $\mathrm{p}=0.65$ and $\mathrm{p}=0.75$, respectively). A meagre reduction in the Ashworth tone scale was observed between the two groups. The adult brain can regenerate neurons lost after brain ischemia. Repair mechanisms in stroke are related to acute injury (first epoch) and is said to occur in the initial few hours after acute event when changes in blood flow, metabolism and ischemic cascade are most active. A second epoch is related with upregulation of growth factors which continue for days to weeks and is referred to as endogenous repair related events. A third epoch occurs weeks to months after stroke when spontaneous recovery mechanisms plateau representing a stable but modifiable early and late chronic phase. The purpose of this study was to attain maximum restoration possible and eventual return to normalcy of function. We followed up those six patients till four years. This short article presents safety, feasibility and tolerance of bone marrow derived mesenchymal stem cells after four years of infusion. It also studies the efficacy end points in terms of clinical and laboratory parameters at the end of 208 weeks (4 years).

\section{Methods}

Twelve patients $(\mathrm{n}=12)$ diagnosed with stroke (index event) 3 months to 2 years, MRC (Medical Research Council) grade of muscle power for the wrist and hand extensor or flexor muscles of at least 2, NIHSS (National Institute of Health Stroke Scale) between 4 and 15 who were conscious and comprehendible were recruited. All the patients were assessed on muscle power (MRC), tone (modified Ashworth) and Fugl Meyer (FM) scale for upper limbs and modified Barthel index (mBI) at baseline, 8weeks, 24, 78, 156 and 208 weeks $^{[14,15]}$. The study was approved by Institute Committee for Stem Cell Research and Therapy (ICSCRT) and written informed consent was obtained from all the subjects. The trial is registered with CTRI (Ref/2011/08/002677).

\section{Procedure}

\section{Bone Marrow Aspiration, Expansion and Transplantation}

Bone marrow was aspirated under aseptic conditions from the posterior superior iliac crest of 6 chronic stroke patients. The bone marrow was diluted with phosphate-buffered saline, layered over Ficoll density medium and centrifuged at 1,800 rpm. for $25 \mathrm{~min}$. Using Stem Pro MSC SFM basal medium (A-10334, Invitrogen), the mononuclear cell were plated at a density of $10^{6}$ cells $/ \mathrm{cm}^{2}$ in T-25 tissue culture flask and incubated at $37^{\circ} \mathrm{C} / 5 \% \mathrm{CO}^{2}$. The cells were harvested and seeded at 3,000 or 10,000 cells $/ \mathrm{cm}^{2}$ in triplicate wells of 6-well plates. Trypan blue dye was used to test viability. MSC cultures of all patients were harvested using TrypLE TM Express (Invitrogen) on reaching 70-90\% confluency. Non-adherent cells were removed after $24 \mathrm{~h}$ and fresh media was again added for incubation $^{[16,17]}$. The whole procedure took around $21 \pm 7$ days. All samples were tested for mycoplasma and endotoxins at every third passage using commercially available kits according to the manufacturer's instructions. Aseptic infusion technique was followed and cells were directly dissolved in 250-ml saline bottle and infused intravenously over $2-3 \mathrm{~h}$ using a sterile $50-\mathrm{ml}$ syringe. The control group was administered with neurophysiotherapy regime only for 8 weeks.

\section{Statistics}

Mean difference between groups was compared using t test. We used both parametric and non-parametric tests for the outcome measures. Mortality was defined as death of a subject enrolled in the study till the end point which includes all cause mortality (cardiac, non cardiac and vascular) and the survival time was the day the patient received stem cell transplantation till the follow up or the end of study. Individual changes in FM and $\mathrm{mBI}$ between baseline and 8, 78, 156 weeks and 208 weeks were analyzed by parametric $t$ test. A p $<0.05$ was taken to indicate statistical significance. All statistical analyses were conducted using SPSS 12.0 (Chicago, IL).

\section{Result}

\section{Baseline Characteristics of Patients}

A total of twelve $(n=12)$ patients were included in this study allocated to both the groups. Their mean age was $42.8 \pm 16.4$ years $($ mean $\pm \mathrm{SD})$. The laboratory tests after the stem cell transplantation were normal for all patients as described till the last follow up.. The mean cell viability at transplantation was $98 \%$, the cells were sterile and endotoxin free during expansion and at the time of injection. There were no early and late adverse reactions observed in patients during and after transplantation. Flow-cytometric analysis showed phenotype markers such as CD90, CD73, CD105 and were negative for HLA class II. The mean CD90, CD73 and CD105 were 61, 57.1 and $40 \%$, respectively.

\section{Mortality and adverse events}

The IRB approved only six patients for MSC transplantation as pilot study in the year 2010, none of the patients reported malignancies, tumor or any cystic malformations when examined at fours years. These patients were screened with regular clinical examination, laboratory and radiology (i.e., MRI scans) investigations. They were telephonically interviewed (if it was not possible for them for a hospital visit) for any delayed reactions of skin, untreated infections or any other alarming symptoms. One of the patient (id 6) reported skin allergy/ rash after 9 months of transplantation (table 2). $\mathrm{He}$ underwent hospitalization for the same but it was found that the infection was unrelated to cells. Recurrent stroke or TIAs were not reported in all the six subjects.

\section{Clinical Results}

In the MSC or experimental group (males: females = 2:4), all were right handed dominant with age $=42 \pm 16.4$ years $($ mean \pm SD); the mean FM score was $44 \pm 11.6$ at baseline and $53 \pm 7.1$ at $208^{\text {th }}$ week $(\mathrm{p}=0.026, \mathrm{t}=-2.26)$, at 78 weeks $(45.5 \pm 7.2)$ exhibiting statistically significant improvement between all time points $(\mathrm{p}<0.05)$ (table 1$)$. The second quartile for the experimental group for $\mathrm{mBI}$ and $\mathrm{FM}$ at 208 weeks was 92 and 54 respectively whereas control group showed a median of 51.7 and 47.4 respectively. Repeated measures ANOVA was found to be statistically significant at all time measurements i.e., at baseline and at 8, 24 weeks 78, 156 and 208 weeks ( $<<0.05)$. The control group showed significant improvement also between baseline and four year assessment. The mean FM scores at baseline was $16.8 \pm 6.1$ and at 208 weeks was $48 \pm 5.2(\mathrm{p}=0.002)$ respectively. These patients also showed statistically significant improvement between baseline and 24, 24 and 78, 156 to 208 weeks $(\mathrm{p} \leq 0.05)$ for both FM and $\mathrm{mBI}$ scores. 
Table 1. Clinical outcomes and demographics in experimental / (MSC) and control group

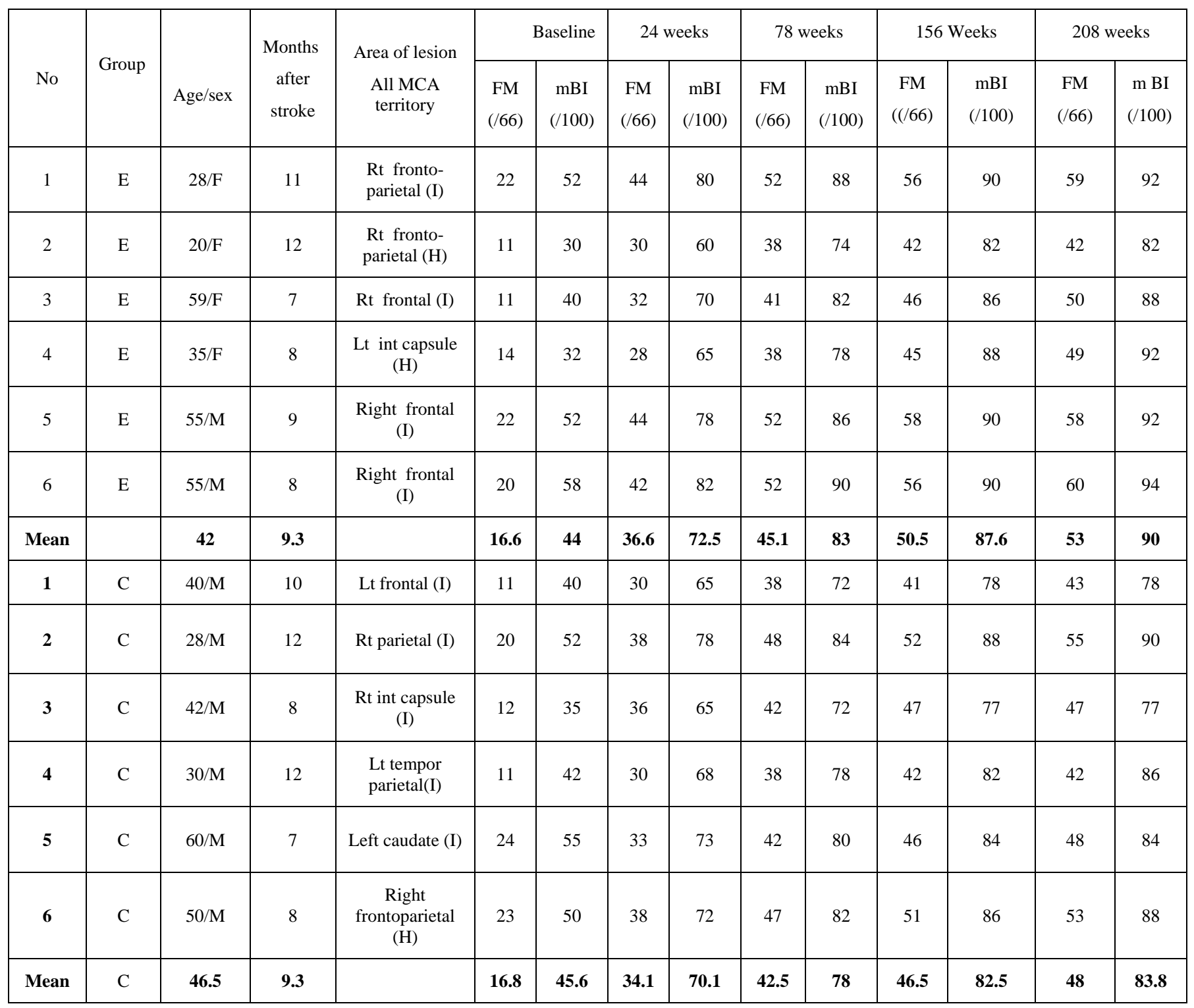

Table 2. Onsetof morbidities and reactions in both the groups. None were related to cell transplantation

\begin{tabular}{|c|c|c|}
\hline Morbidities and reactions & MSC group & Control group \\
\hline Early reactions & 1 & 0 \\
\hline Fever & 0 & 0 \\
\hline Infection (pneumonia,UTI etc) & 2 & 0 \\
\hline Pain & 0 & 1 \\
\hline drowziness & 0 & 0 \\
\hline Long term effects & 0 & 0 \\
\hline Tumor formation & 0 & 0 \\
\hline Systemic cancer & 1 & 2 \\
\hline Brain tumor & 1 & 2 \\
\hline Seizures & & \\
\hline Psychological illnes & 0 & \\
\hline
\end{tabular}




\section{Comparison between MSC and Control Group}

The baseline characteristics between the two groups were matched ( $>0.05$ ) suggesting that the two groups were comparable to study the effectiveness of therapy after 8, 24, 78, 156 and 208 weeks. Only mBI was statistically significant at 208 weeks (95 \% CI: -12.9 to 0.49 ; $\mathrm{p}=0.05$ ) and at 156 weeks (95\% CI: -1.26 to 1.76 ; $\mathrm{p}=0.04$ ) (figures $1 \&$ 2) whereas there was no significant difference in FM scores at four yearly examination (95\% CI:-3.01 to 2.01, $\mathrm{p}=0.19$ ). The estimated difference between the two proportions for Fugl Meyer scale was 5 at 4 years. We did not report any significant improvement in power and tone measured on MRC and Ashworth tone scale for upper and lower limbs.

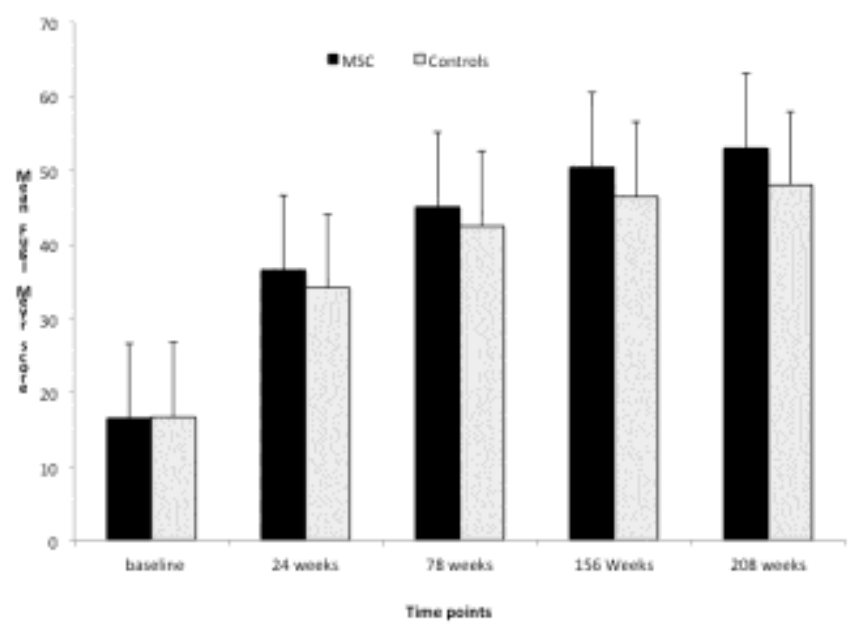

Figure 1. Graph showing mean Fugl Meyer scores between experimental and control groups at all time points.

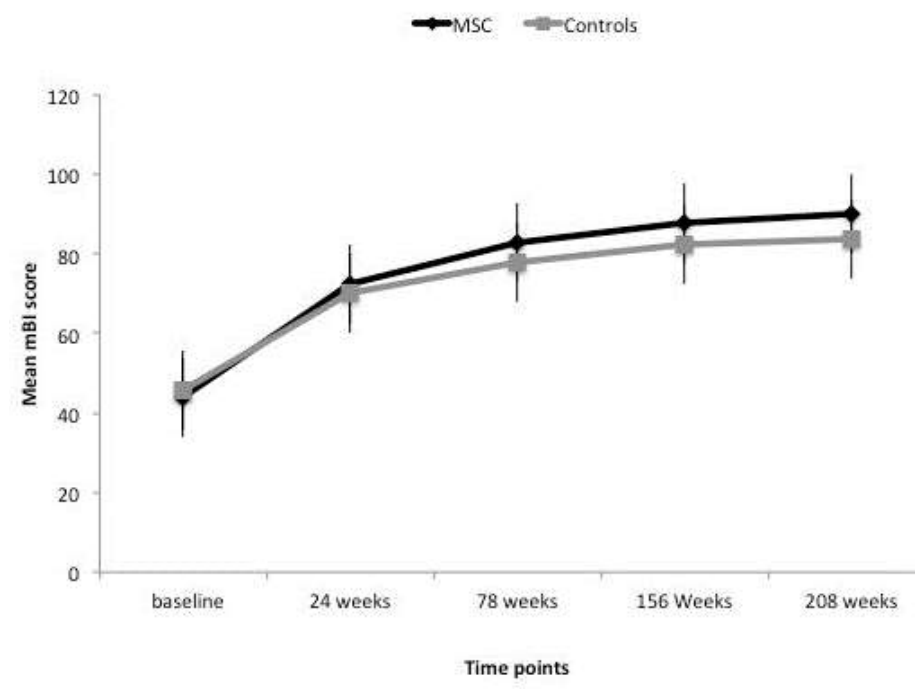

Figure 2. Graph showing mean modified Barthel Index in experimental and control groups at all time points.

\section{Discussion}

We had studied safety and feasibility of ex vivo culture expanded mesenchymal stem cells with animal serum free media in our published trials ${ }^{[13,18,19]}$. Owing to ethical concerns of using these cells, it was necessary to monitor these patients for more safety concerns and potential late complications i.e., tumorogenesis and genetic mutations. Our first study was morally based on the safety, feasibility, practicality and procurement of mesenchymal cells in which we did not report any significant improvement in the functional outcomes till 24 weeks. In this report, we present safety and efficacy of mesenchymal stem cells at 208 weeks (4years) in six chronic stroke patients. In this mid- to long-term follow up, we present only the clinical data and not the functional imaging results, as two of the patients could not undergo MRI due to claustrophobia and hence BOLD and DTI results are not explained in this.

Bang et al in their 5 year follow up ${ }^{[21]}$ reported the risk of zoonoses raising the possibility that administration of MSCs contaminated by xenogeneic proteins lead to a risk of immunologic rejection of the injected cells and serious complications such as autoimmune reactions against one's own stem cells ${ }^{[22,23]}$. As we used no animal medium for the culture expansion so the risk of occurence of GVHD was not expected and hence not reported in our patients.

The results of the present study showed that intravenous transplantation of ex vivo culture expanded MSCs is safe based on results of four-years of follow up. There was no mortality or any comorbidities during this period. Supraventricular tachyarrhythmia was reported in patients who received hematopoietic stem cell transplantation. Similarly, it is hypothesized that seizures may be caused by aberrant innervation from newly formed neural circuits after cell transplantation. We did not report any such incidence in our patients. In addition, it is also reported that, vascular occlusion can be caused by MSCs at the time of infusion via occlusion of the arteries of the brain or other organs or by restenosis ${ }^{[24]}$. Fortunately, our six patients tolerated the transplantation well and are still under longterm follow-up for any such event occurence. In our study no immune suppressants were required following transplantation, eliminating the risks associated with MSC therapy ${ }^{[25-27]}$. This is the first known study with a relatively long term follow up establishing the safety and tolerance of MSC derived using serum-free media for expansion unlike bovine serum used in the earlier study ${ }^{[21]}$.

A score of 92 i.e., $75^{\text {th }}$ percentile of $\mathrm{mBI}$ in experimental group as compared to 85 in control reflects a 7 point difference suggesting that control group also recovered well. In our published study, we presented that intravenous delivery of cells as safe, well tolerated by patients till 6 months but we failed to prove any remarkable difference between the two groups. A four-year long follow up can be considered a good reference to scale stroke recovery, very likely the patients must have opted for alternative systems of medicine i.e., acupuncture, acupressure, naturopathy and other uncontrolled physiotherapy regimes. Till 24 weeks as last reported, there was no improvement in the activities of daily living scale whereas when examined at 208 weeks, patients performed functionally better in the MSC group than in the control group with modified Barthel index being statistically significant $(\mathrm{p}<0.05)$. Enhancing neurogenesis can be a candidate explanation of the therapeutic mechanism of MSCs. Preclinical studies showed the importance of neurogenesis in an animal model of stroke and transplanted MSCs might enhance this process $^{[28,29]}$. It has been proven that stem cells home in the infarcted regions thus promoting functional recovery in chronic stroke rats $^{[30,31]}$. As reported earlier, cell-enhanced recovery has been reported with chronic delivery of cells even 1 month after ischemia $^{[32]}$.

We did not observe any significant improvement in MRC, Ashworth tone scale, Fugl Meyer and volume of lesion at four years between the two groups. The recovery observed was in aspects of functional gains although it was reported from patients with stem cell therapy that their performance and task oriented activity had increased compared to the pre stem cell status. Owing to ethical concerns and 
rampant use of stem cells without regulation, the institute ethics committee gave approval for six patients initially. The control group also showed improvement with an increased clinical and ADL scores which would question the psychoimmunological or placebo effects of mesenchymal stem cells.

The interval between the onset of stroke and the time of cell therapy may be an important criteria for selecting patients for cell therapy. Stroke is classically divided into an acute, sub-acute and chronic phase. Each phase is defined by a complex array of events with overlapping and distinct kinetics that led to tissue regeneration and remodelling. Cell transplantation at a chronic phase is thought to augment the neurobehavioural responses after injury via neurotrophic approach. The homogeneity of the subject group i.e., the type of stroke, etiology, premorbid status are few questions which need to be answered and stated before planning a trial ${ }^{[33,34]}$. Recently, there have been various efforts to enhance the therapeutic beneficial effects of stem cells (including blood-brain barrier manipulation, chronic preconditioning and genetically modified MSCs) and to reduce possible adverse effects of MSCs. We hope the therapeutic effects and safety of MSCs will be improved with these efforts and further studies.

The Indian government has regularized stem cell research practices in the country with the premier research institutes like Indian council of Medical Research and the Department of Biotechnology having laid down stem cell research guidelines in 2013 according to which all clinical trials in India should be approved by IC-SCRT and national apex committee (NAC) along with CDSCO draft on compensation towards injury due to participation in clinical research ${ }^{[35,36]}$. Phase II of a randomized controlled transplantation of autologous stem cells in stroke by our group is currently undergoing which would help to delineate the efficacy of these cells in a more scientific manner and amend the pitfalls in the current study. A very recent study published by Prasad et al $^{[37]}$ investigated the safety and efficacy of intravenous mononuclear stem cell transplantation in sub-acute ischemic stroke in which fifty-eight patients received a mean of 280.75 million BMSCs at median of 18.5 days after stroke onset. There was no significant difference between BMSCs arm and control arm in the Barthel Index score (63.1 versus 63.6; $\mathrm{p}=0.92$ ), modified Rankin scale shift analysis $(\mathrm{p}=0.53)$ or score $>3(47.5 \%$ versus $49.2 \% ; \mathrm{p}=0.85)$, NIHSS score ( 6.3 versus $7.0 ; p=0.53$ ) which is similar to our study as there was no statistically significant improvement observed between study and control group.

\section{Conclusion}

As no study is flawless, we also faced several limitations which require mention. First, owing to the experimental nature of treatment, the sample size was very small to state the efficacy of the cells $^{[32,33]}$. Altogether all current human studies imply that stem cell therapy in brain is feasible, the limited data obtained from this research thus far provide little consistent evidence of any clinical benefit. Further trials are needed to determine the optimal cell population and method of administration is needed to improve the outcome of cell therapy for stroke ${ }^{[38,39]}$. The results of the present study showed some potential of MSC in regard to the functional improvement in patients nevertheless the trial primarily explains the tolerance, forbearance and safety associated with MSCs transplantation widening their scope for translational medicine.

\section{References}

1. Eriksson PS, Perfilieva E, Bjork-Eriksson T. Neurogenesis in the adult human hippocampus. Nat Med. 1998; 4(11): 1313-7.

2. Stahnisch FW, Nitsch R. Cajal's concept of neuronal plasticity: the ambiguity lives on. Trends Neurosci. 2002; 25(11): 589-91.
3. Lee JS, Hong JM, Moong GJ, Lee PH, Bang OY. Long-Term Effects of MSC Treatment in Stroke. Stem Cells. 2010; 28(6):1099-106.

4. Bjorklund A, Lindvall O. Cell replacement therapies for central nervous system disorders. Nat Neurosci. 2000; 3(6):537-44.

5. Weimann JM, Charlton CA, Brazelton TR, Hackman RC, Blau HM. Contribution of transplanted bone marrow cells to purkinje neurons in human adult brains. Proc Natl Acad Sci USA. 2003; 100(4):2088-93.

6. Sigrid C, Schwarz J. Translation of stem cell therapy for neurological diseases. Translational Research. 2010; 156(3): 15560.

7. Gogel S, Gubernator M, Minger SL. Progress and prospects: stem cells and neurological diseases. Gene Ther. 2011;18(1):1-6

8. Chen J, Chopp M. Neurorestorative treatment of stroke: cell and pharmacological approaches. NeuroRx. 2006;3(4):466-73.

9. Prockop DJ. Marrow stromal cells as stem cells for non hematopoietic tissues. Science. 1997;276(5309):71-4.

10. Conget PA, JJ Minguell. Phenotypical and functional properties of human bone marrow mesenchymal progenitor cells. J Cell Physiol. 1999;181(1):67-73.

11. Caplan AI. Why are MSCs therapeutic? New data: new insight. J Pathol. 2009;217(2):318-24.

12. Castro Henrique P, Coehlo Pimentel P, Fonseca M, Freitas GR, Otero RM. The rise of Cell Therapy trials of Stroke. Review of Registered and published studies. Stem Cells Dev. 2013 ;22(15):2095-111.

13. Bhasin A, Srivastava M, Mohanty S, Kumaran S, Bhatia R, Garg A, Airan B. Autologouss mesenchymal stem, cells in chronic stroke. Cerebrovasc Dis Extra. 2011;1(1):93-104.

14. Loewen SC, Anderson BA: Reliability of modified motor assessment scale and the Barthel index. Phys Ther. 1988;68(7):1077-81.

15. Oldfield RC: The assessment and analysis of handedness: the Edinburgh inventory. Neuropsychologia. 1971;9(1):97-113.

16. Chase LG, Lakshmipathy U, Rao SM, Vemuri MC. A novel serum-free medium for the expansion of human mesenchymal stem cells. Stem Cell Res Ther. 2010;1(1):8.

17. Lindroos B, Boucher S, Chase L, Kuokkanen H, Huhtala H, Haataja R. Serum-free, xeno-free culture media maintain the proliferation rate and multipotentiality of adipose stem cells in vitro. Cytotherapy. 2009;11(7):958-72.

18. Bhasin A, Srivastava MVP, Bhatia R, S Mohanty S, Kumaran S, Bose S. Stem cell therapy. A clinical trial in Stroke. Clin Neurol Neurosurg. 2013;115(7):1003-8.

19. Bhasin A, Srivastava MVP, Bhatia R, Mohanty S, Senthil S. Autologous Intravenous mononuclear stem cell therapy in chronic ischemic stroke. J Stem Cells Regen Med. 2012;8(3):181-9.

20. Wei X, Yang X, Han ZP, Qu F, Shao L, Shi Y. Mesenchymal stem cells: a new trend for cell therapy. Acta Pharmacol Sin. 2013;34(6):747-54.

21. Bang OY, Lee JS, Lee PH, Lee G. Autologous mesenchymal stem cell transplantation in stroke patients. Ann Neurol. 2005;57(6):874-82.

22. Spees JL, Gregory CA, Singh H, Tucker HA, Peister J, Lynch PJ, Hsu SC, Smith J, Prockop DJ. Internalized antigens must be removed to prepare hypoimmunogenic mesenchymal stem cells for cell and gene therapy. Mol Ther. 2004;9(5):747-56.

23. Cobo F, Talavera P, Concha A. Diagnostic approaches for viruses and prions in stem cell banks. Virology. 2006;347(1):1-10.

24. Hidalgo JD, Krone R, Rich MW, Blum K, Adkins D, Fan MY, Brown R, Devine S, Grawbert T, Blum W, Tomasson M, Goodnough LT, Vij R, Dipersio J, Khoury H. Supraventricular tachyarrhythmias after hematopoietic stem cell transplantation: Incidence, risk factors and outcomes. Bone Marrow Transplant. 2004;34(7):615-9.

25. Aggarwal S, Pittenger MF. Human mesenchymal stem cells modulate allogeneic immune cell responses. Blood. 2005;105(4):1815-22.

26. Zhang R, Zhang Z, Wang L, Wang Y, Gousev A, Zhang L, Ho KL, Morshead C, Chopp M. Activated neural stem cells contribute to stroke-induced neurogenesis and neuroblast migration toward the infarct boundary in adult rats. J Cereb Blood Flow Metab. 2004;24(4):441-8. 
27. Kokaia Z, Thored P, Arvidsson A, Lindvall L. Regulation of stroke-induced neurogenesis in adult brain-recent scientific progress. Cereb Cortex. 2006;16 Suppl 162-7.

28. Jin K, Wang X, Xie L, Mao XO, Zhu W, Wang Y, Shen J, Mao Y, Banwait S, Greenberg DA. Evidence for stroke-induced neurogenesis in the human brain. Proc Natl Acad Sci U S A. 2006 ;103(35):13198-202.

29. Willing A, Lixian J, Milliken M, Poulus S, Zigova T, Song S, Hart C, Sanchoz- Ramos J, Sanberg PR. Intravenous versus intrastriatal cord blood administration in a rodent model of stroke. J Neurosci Res. 2003;73(3):296-307.

30. Shen LH, Li Y, Chen J, Zacharek A, Gao Q, Kapke A, Lu M, Raginski K, Vangpuri P, Smith A, Chopp M. Therapeutic benefit of bone marrow stromal cells administered 1 month after stroke J Cereb Blood Flow Metab. 2007;27(1):6-13.

31. Chen J, Zhang ZG, Li Y, Wang L, Xu YX, Gautam SC, Lu M, Zhu $\mathrm{Z}$, Chopp M. Intravenous administration of human bone marrow stromal cells induces angiogenesis in the ischemic boundary zone after stroke in rats. Circ Res. 2003;92(6):692-9.

32. Shen LH, Li Y, Chen J, Cui Y, Zhang C, Kapke A, Lu M, SavantBhonsale M, Chopp M. One-year follow-up after bone marrow stromal cell treatment in middle-aged female rats with stroke. Stroke. 2007;38(7):2150-6.

33. Freeman TB, Vawter DE, Leaverton PE, Godbold JH, Hauser RA, Goetz CG, Olanow CW. Use of placebo surgery in controlled trials of a cellular-based therapy for parkinson's disease. N Engl J Med. 1999;341(13):988-92.

34. Lindvall O, Kokaia Z, Martinez-Serrano A. Stem cell therapy for human neurodegenerative disorders-how to make it work. Nat Med. 2004;10 Suppl:S42-50.

35. Mannello F, Tonti GA. Concise review: No breakthroughs for human mesenchymal and embryonic stem cell culture: Conditioned medium feeder layer, or feeder-free; medium with fetal calf serum, human serum, or enriched plasma; serum-free, serum replacement nonconditioned medium, or ad hoc formula? Stem Cells. 2007;25(7):1603-9.

36. Savitz SI, Rosenbaum DM, Dinsmore JH,Wechsler LR, Caplan LR. Cell transplantation for stroke. Ann Neurol. 2002;52(3):26675 .

37. Prasad K, Sharma A, Garg A, Mohanty S, Bhatnagar S, Johri S, Singh KK, Nair V, Sarkar RS, Gorthi SP, Hassan KM, Prabhakar S, Marwaha N, Khandelwal N, Misra UK, Kalita J, Nityanand S; INVEST study group. Intravenous autologous mononuclear stem cell therapy for ischemic stroke: a multicentric randomized trial. Stroke. 2014;45(12):3618-24.

38. Mittal S. Stem cell research. India Perspective. Perspect Clin Res. 2013;4(1):105-7.

39. Bhasin A, Sriavstava MV, Bhatia R, Kumaran S, Mohanty S. Stem cells in neurological diseases. Indian Perspective. J Of Stem Cell Res and Therapy. 2014; 4: 3-10.

\author{
Abbreviations \\ MSC: Mesenchymal Stem cells \\ mBI : $\quad$ modified Barthel Index \\ FM : $\quad$ Fugl Meyer scale \\ DTI : Diffusion tensor imaging \\ MRC : Medical Research Council scale for power assessment
}

\title{
Potential Conflicts of Interests
}

None

\section{Acknowledgments}

We acknowledge the Department of Science and Technology, India for the grant in aid support.

\section{Corresponding Author}

M V Padma Srivastava, Department of Neurology, All India Institute of Medical Sciences, New Delhi, India, 110029 vasanthapadma123@gmail.com 